\title{
GAMOW VECTORS IN A PERIODICALLY PERTURBED QUANTUM SYSTEM
}

\author{
M. HUANG
}

\begin{abstract}
We analyze the behavior of the wave function $\psi(x, t)$ for one dimensional time-dependent Hamiltonian $H=-\partial_{x}^{2} \pm 2 \delta(x)(1+2 r \cos \omega t)$ where $\psi(x, 0)$ is compactly supported.

We show that $\psi(x, t)$ has a Borel summable expansion containing finitely many terms of the form $\sum_{n=-\infty}^{\infty} e^{i^{3 / 2}} \sqrt{-\lambda_{k}+n \omega i}|x| A_{k, n} e^{-\lambda_{k} t+n \omega i t}$, where $\lambda_{k}$ represents the associated resonance. This expression defines Gamow vectors and resonances in a rigorous and physically relevant way for all frequencies and amplitudes in a time-dependent model.

For small amplitude $(|r| \ll 1)$ there is one resonance for generic initial conditions. We calculate the position of the resonance and discuss its physical meaning as related to multiphoton ionization. We give qualitative theoretical results as well as numerical calculations in the general case.
\end{abstract}

\section{INTRODUCTION}

Gamow vectors and resonances, introduced by Gamow to describe $\alpha$-decay (cf. [1]), are very important mathematical tools in the study of metastable (or quasistable) states in quantum mechanics (cf. 2]). The decay states described by Gamow vectors are also linked to the Fermi-Dirac golden rule (cf. 9]). There are numerous definitions of resonances and resonant states, using the scattering matrix, rigged Hilbert spaces, Green's function, etc. (cf. 9, 10, and the references therein) These definitions rely on the time-independent Schrödinger equation, though they may be extended to time-dependent settings in a perturbative regime (cf. [3, 14]).

In a recent paper [15, the author and his collaborator gave a rigorous definition of Gamow vectors and resonances for compactly supported time-independent potentials in one dimension, using Borel summation (for a detailed description of Borel summation, see [15, 8]). In this paper, we study the resonances associated to a time-dependent periodic potential. In our case, the Gamow vector is of the form of the so-called Floquet ansatz (cf. 4). Our result holds for all amplitudes and frequencies of the time-dependent field. In the case of small amplitude or high frequency, we calculate the resonances asymptotically, and the real part of the resonances measures the ionization rate. In this sense, our paper extends the results of [5, 16]. As we will see, time dependency introduces new subtleties and complex phenomena.

\section{Setting and Main Results}

We consider the time-dependent one-dimensional Schrödinger equation

$$
i \hbar \frac{\partial}{\partial t} \psi(x, t)=-\frac{\hbar}{2 m} \frac{\partial^{2}}{\partial x^{2}} \psi(x, t)+V(x, t) \psi(x, t)
$$


where the potential $V(x, t)$ is a delta function potential well or barrier with a timeperiodic perturbation. In this paper, we consider two simple but illuminating cases:

(1) delta potential well $V(x, t)=-2 A \delta(x)(1+2 r \cos \omega t)$

(2) delta potential barrier $V(x, t)=2 A \delta(x)(1+2 r \cos \omega t)$

Here $A>0$ represents the strength of the potential, $r$ represents the relative amplitude of the perturbation and $\omega$ the frequency. Without loss of generality we take $r>0, \omega>0$. We further assume the initial wave function $\psi_{0}(x):=\psi(x, 0)$ is compactly supported and $C^{2}$ on its support.

We first normalize the equation by changing variables $x \rightarrow \frac{\hbar}{2 m A} x, t \rightarrow \frac{\hbar^{2}}{2 m A^{2}} t, \omega \rightarrow$ $\frac{2 m A^{2}}{\hbar^{2}} \omega$. Note that this is more than using atomic units since we also used the special property of the delta function $\delta(A x)=\delta(x) / A$. The equation becomes

$$
i \frac{\partial}{\partial t} \psi(x, t)=-\frac{\partial^{2}}{\partial x^{2}} \psi(x, t) \mp 2 \delta(x)(1+r \cos \omega t) \psi(x, t)
$$

(where "-" corresponds to the delta potential well and "+" corresponds to the barrier) We shall focus on the delta potential well and analyze in detail the behavior of the wave function as well as the resonances of the system for all amplitudes and frequencies. The analysis of the delta potential barrier is very similar and we will give the results in Section 4 without detailed proofs.

Theorem 1. Assume the initial wave function $\psi(x, 0)$ is compactly supported and $C^{2}$ on its support, then we have for all $t>0$

$$
\begin{gathered}
\psi(x, t)=\sum_{k=1}^{K} \sum_{n=-\infty}^{\infty} e^{i^{3 / 2} \sqrt{-\lambda_{k}+n \omega i}|x|} A_{k, n} e^{-\lambda_{k} t+n \omega i t} \\
-\frac{1}{2 \pi i} \sum_{n=-\infty}^{\infty} \int_{0}^{e^{i \theta} \infty} e^{i^{3 / 2} \sqrt{-q+n \omega i}|x|+n \omega i t-q t} \varphi_{n}(-q) d q-\frac{1}{2 \pi i} \int_{0}^{e^{i \theta} \infty} F(x,-q) e^{-q t} d q
\end{gathered}
$$

where $\lambda_{k}+n \omega i$ are resonances of the system $\left(\operatorname{Re}\left(\lambda_{k}\right)>0\right), \varphi$ a ramified analytic function with square root branch points at every $n \omega i(n \in \mathbb{Z})$, and $F$ an explicit function with $\sqrt{p} F(p)$ analytic in $\sqrt{p}$. $\theta$ is a small angle chosen to ensure that no resonance lies on the path of integration.

Moreover, the coefficients $A_{k, n}$ satisfy the recurrence relation

$$
\left(\sqrt{-i} \sqrt{i+n \omega i-\lambda_{k}}-1\right) A_{k, n}=r A_{k, n-1}+r A_{k, n+1}
$$

and $\psi(x, t)$ has the Borel summable representation

$$
\begin{aligned}
\psi(x, t)=\left(i^{3 / 2} r \int_{-\infty}^{\infty} \psi_{0}(x) d x\right) t^{-1 / 2} & +\sum_{n=-\infty}^{\infty} \sum_{k=0}^{\infty} C_{n, k}(x) e^{n \omega i t} t^{-3 / 2-k} \\
& +\sum_{k=1}^{K} \sum_{n=-\infty}^{\infty} e^{i^{3 / 2} \sqrt{-\lambda_{k}+n \omega i}|x|} A_{k, n} e^{-\lambda_{k} t+n \omega i t}
\end{aligned}
$$

Corollary 1. For $1 \leqslant k \leqslant K$, the Gamow vector term

$$
\sum_{n=-\infty}^{\infty} e^{i^{3 / 2} \sqrt{-\lambda_{k}+n \omega i}|x|} A_{k, n} e^{-\lambda_{k} t+n \omega i t}
$$


is a generalized eigenvector of the Hamiltonian, in the sense that it solves (1), but grows exponentially (in a prescribed fashion) for large $|x|$.

Proposition 2. For small $r$ there is only one array of resonances, i.e. $K=1$. The asymptotic position of the array of resonances and a similar result for large $\omega$ are given in Section 3.4

In the above formulas the branch of the square root is chosen to be the usual one: $\arg (z) \in(-\pi, \pi]$ and $\arg (\sqrt{z}) \in\left(-\frac{\pi}{2}, \frac{\pi}{2}\right]$. We refer to this choice of branch when we use the phrase "usual (choice of) branch" in this paper.

For small $r$ we calculate asymptotically the position of the resonance, which is related to the ionization rate. For generic $r$ we will give numerical results showing that the Gamow vector terms exist for some but not all $r$, and we plot the graph of the positions of resonances with different amplitudes (see Section 4).

Remark 1. Theorem 1 and its corollaries generalize to the case where

$$
V(x, t)=\mp 2 A \delta(x)\left(1+2 \sum_{k=1}^{K_{0}}\left(r_{k} \cos k \omega t+s_{k} \sin k \omega t\right)\right)
$$

\section{Proof of Main Results}

3.1. Integral reformulation of the equation. We first consider the Laplace transform in $t$

$$
\hat{\psi}(x, p)=\int_{0}^{\infty} e^{-p t} \psi(x, t) d t
$$

The existence of this Laplace transform (for $\operatorname{Re}(p)>0$ ) follows from the existence of a strongly differentiable unitary propagator (see Theorem X.71, 6] v.2 pp 290, see also [7, [15] and [16]). As we will see, Theorem 1 follows from analyzing the singularities (poles and branch points) of the analytic continuation of $\hat{\psi}(x, p)$.

Performing this Laplace transform on (1), we obtain

$$
\begin{aligned}
& i p \hat{\psi}(x, p)-i \psi_{0}(x)= \\
& \quad-\frac{\partial^{2}}{\partial x^{2}} \hat{\psi}(x, p)-2 \delta(x) \hat{\psi}(x, p)-2 r \delta(x) \hat{\psi}(x, p-i \omega)-2 r \delta(x) \hat{\psi}(x, p+i \omega)
\end{aligned}
$$

We then rewrite the above ordinary differential equation as an integral equation by inverting the operator $\frac{\partial^{2}}{\partial x^{2}}+i p$. We have

$$
\hat{\psi}(x, p)=\frac{\sqrt{i} e^{-i^{3 / 2} \sqrt{p} x}}{2 \sqrt{p}} \int_{+\infty}^{x} e^{i^{3 / 2} \sqrt{p} s} g(s) d s-\frac{\sqrt{i} e^{i^{3 / 2}} \sqrt{p} x}{2 \sqrt{p}} \int_{-\infty}^{x} e^{-i^{3 / 2} \sqrt{p} s} g(s) d s
$$

where

$$
g(x)=i \psi_{0}(x)-2 \delta(x) \hat{\psi}(x, p)-2 r \delta(x) \hat{\psi}(x, p-i \omega)-2 r \delta(x) \hat{\psi}(x, p+i \omega)
$$

Recalling that $\int_{-\infty}^{\infty} \delta(x) f(x) d x=f(0)$, we simplify the above integral equation and obtain 


$$
\begin{array}{r}
\hat{\psi}(x, p)=\frac{e^{-i^{3 / 2} \sqrt{p} x}}{2 i^{-3 / 2} \sqrt{p}} \int_{+\infty}^{x} e^{i^{3 / 2} \sqrt{p} s} \psi_{0}(s) d s-\frac{e^{i^{3 / 2} \sqrt{p} x}}{2 i^{-3 / 2} \sqrt{p}} \int_{-\infty}^{x} e^{-i^{3 / 2} \sqrt{p} s} \psi_{0}(s) d s \\
+\frac{\sqrt{i} e^{i^{3 / 2} \sqrt{p}|x|}}{\sqrt{p}}(\hat{\psi}(0, p)+r \hat{\psi}(0, p-i \omega)+r \hat{\psi}(0, p+i \omega))
\end{array}
$$

Letting $x=0$ we get an equation for $\hat{\psi}(0, p)$

$$
\begin{aligned}
\hat{\psi}(0, p)=\frac{i^{3 / 2}}{2 \sqrt{p}} \int_{+\infty}^{0} e^{i^{3 / 2} \sqrt{p} s} \psi_{0}(s) d s-\frac{i^{3 / 2}}{2 \sqrt{p}} \int_{-\infty}^{0} e^{-i^{3 / 2} \sqrt{p} s} \psi_{0}(s) d s & \\
+ & \frac{\sqrt{i}}{\sqrt{p}}(\hat{\psi}(0, p)+r \hat{\psi}(0, p-i \omega)+r \psi(0, p+i \omega))
\end{aligned}
$$

which implies

$$
\begin{aligned}
& \frac{\sqrt{i}}{\sqrt{p}}(\hat{\psi}(0, p)+r \hat{\psi}(0, p-i \omega)+r \psi(0, p+i \omega))= \\
& \hat{\psi}(0, p)-\frac{i^{3 / 2}}{2 \sqrt{p}} \int_{+\infty}^{0} e^{i^{3 / 2} \sqrt{p} s} \psi_{0}(s) d s-\frac{i^{3 / 2}}{2 \sqrt{p}} \int_{-\infty}^{0} e^{-i^{3 / 2} \sqrt{p} s} \psi_{0}(s) d s
\end{aligned}
$$

Substituting (6) in (4) we get

$$
\hat{\psi}(x, p) \quad=\quad e^{i^{3 / 2} \sqrt{p}|x|} \hat{\psi}(0, p) \quad+\quad f(x, p) \quad-\quad e^{i^{3 / 2} \sqrt{p}|x|} f(0, p)
$$

where

$$
f(x, p)=\frac{i^{3 / 2} e^{-i^{3 / 2} \sqrt{p} x}}{2 \sqrt{p}} \int_{+\infty}^{x} e^{i^{3 / 2} \sqrt{p} s} \psi_{0}(s) d s-\frac{i^{3 / 2} e^{i^{3 / 2} \sqrt{p} x}}{2 \sqrt{p}} \int_{-\infty}^{x} e^{-i^{3 / 2} \sqrt{p} s} \psi_{0}(s) d s
$$

Equation (17) indicates that the analytic continuation of $\hat{\psi}(x, p)$, as well as its singularities, follows naturally from that of $\hat{\psi}(0, p)$, so it suffices to analyze $\hat{\psi}(0, p)$ using the recurrence relation (5). Later we will perform the inverse Laplace transform on $\hat{\psi}(x, p)$, justified by estimating $\hat{\psi}(0, p)$ and $f(x, p)$ for large $p$. We will then deform the contour of the Bromwich integral, which yields the expression in Theorem 1. It is worth noting that to deform the contour it suffices to place a branch cut of the square root in the left half complex plane, while to analyze the singularities of $\hat{\psi}(0, p)$ we need to consider a larger region in the Riemann surface. Some delicate points of the analysis stems from the complexity of the Riemann surface, since, as we will see, $\hat{\psi}(0, p)$ has infinitely many branch points and there appears to be a barrier of singularities on the non-principal Riemann sheet.

3.2. Recurrence relation and analyticity of $\hat{\psi}$. We rewrite the recurrence relation (5) as

$$
(\sqrt{-i} \sqrt{p}-1) \hat{\psi}(0, p)=r \hat{\psi}(0, p-i \omega)+r \hat{\psi}(0, p+i \omega)+\sqrt{-i} \sqrt{p} f(0, p)
$$

We will show that $f(0, p)=\frac{\psi_{0}(0)}{p}+O\left(\frac{1}{p^{3 / 2}}\right)$ as $p \rightarrow \infty$ in any direction in the right half complex plane (see Section [3.6). It is not a priori clear that $\hat{\psi}(0, p)$ has 
an inverse Laplace transform. We thus let $\tilde{\psi}(p)=\hat{\psi}(0, p)-f(0, p)$. The recurrence relation for $\tilde{\psi}$ is

$$
(\sqrt{-i} \sqrt{p}-1) \tilde{\psi}(p)=r \tilde{\psi}(p-i \omega)+r \tilde{\psi}(p+i \omega)+(1+2 r) f(0, p)
$$

It is convenient to write the recurrence relation in a difference equation form. Denoting $p=i+i n \omega+z, y_{n}(z)=\tilde{\psi}(i+i n \omega+z)$, and $f_{n}(z)=(1+2 r) f(0, i+i n \omega+z)$, we have

$$
(\sqrt{-i} \sqrt{i+i n \omega+z}-1) y_{n}(z)=r y_{n-1}(z)+r y_{n+1}(z)+f_{n}(z)
$$

The associated homogeneous equation is of course

$$
(\sqrt{-i} \sqrt{i+i n \omega+z}-1) y_{n}(z)=r y_{n-1}(z)+r y_{n+1}(z)
$$

Let $z_{0}$ be a branch point closest to 0 , that is, a point on the imaginary axis satisfying $-z_{0} i=\inf _{n}\{|1+n \omega|\}$ (note that $\left|z_{0}\right| \leqslant \frac{\omega}{2}$ ), and let $n_{0}$ be the corresponding $n$. Since clearly $y_{n}(z)=y_{n+1}(z-i \omega)=y_{n-1}(z+i \omega)$, it suffices to consider $\operatorname{Im}(z) \in\left(-\frac{4}{5} \omega, \frac{4}{5} \omega\right)$ for the usual branch. In general, if we make a branch cut at $\left(e^{i \theta} \infty, z_{0}\right)(\cos \theta \neq 0)$ we consider the strip-shaped region $\{|\operatorname{Im}(z)-\rho \sin \theta|<$ $\left.\frac{4}{5} \omega, \operatorname{Re}(\mathrm{z})=\rho \cos \theta, \rho \in \mathbb{R}\right\}$.

To analytically continue $\mathbf{y}:=\left\{y_{n}\right\}$, we consider the Hilbert space $\mathcal{H}$ defined by

$$
\|\mathbf{x}\|_{\mathcal{H}}^{2}=\sum_{n=-\infty}^{\infty}\left(1+|n|^{3 / 2}\right)\left|x_{n}\right|^{2}
$$

and the operator $\mathcal{C}_{m}: \mathcal{H} \rightarrow \mathcal{H}$

$$
\left(\mathcal{C}_{m} \mathbf{y}\right)_{n}(z)=\frac{(1+m \sqrt{i}) y_{n}(z)+r y_{n-1}(z)+r y_{n+1}(z)}{(\sqrt{-i} \sqrt{i+i n \omega+z}+m \sqrt{i})}\left(m \in \mathbb{Z}^{+}\right)
$$

It is easy to see that $\mathcal{C}_{m}$ is entire in $r$ and analytic in $\sqrt{z-z_{0}}$ in the region $\operatorname{Re}(z)>-m^{2}, \operatorname{Im}(z) \in\left(-\frac{4}{5} \omega, \frac{4}{5} \omega\right)$.

Lemma 3. $\mathcal{C}_{m}$ is a compact operator for any choice of branch.

Proof. For arbitrarily large $N \in \mathbb{N}$, we consider the finite rank operator $\mathcal{D}_{m, N}$ : $\mathcal{H} \rightarrow \mathcal{H}$

$$
\left(\mathcal{D}_{m, N} \mathbf{y}\right)_{n}= \begin{cases}\left(\mathcal{C}_{m} \mathbf{y}\right)_{n} & |n|<N \\ 0 & \text { otherwise }\end{cases}
$$

It is easy to check that

$$
\left\|\mathcal{C}_{m}-\mathcal{D}_{m, N}\right\|=O\left(N^{-1 / 2}\right)
$$

Therefore $\mathcal{C}_{m}$, being the limit of finite rank operators in operator norm, is compact.

Lemma 4. The equation

$$
(\sqrt{-i} \sqrt{i+i n \omega+z}-1) y_{n}(z)=r y_{n-1}(z)+r y_{n+1}(z)+g_{n}(z)
$$


has a unique solution in $\mathcal{H}$ for $|\operatorname{Re}(z)|>(2 r+1)^{2}$, for all $\mathbf{g} \in \mathcal{H}$. In particular, (9) has a unique solution and (10) has only the trivial solution $y=0$. The conclusion holds as well if $z \neq 0$ and $r$ is sufficiently small. Furthermore, for large $|\operatorname{Re}(z)|$ we have $|\mathbf{y}|=O\left(|\operatorname{Re}(z)|^{-1 / 2}|\mathbf{g}|\right)$ where $|\mathbf{x}|:=\sup _{n}\left|x_{n}\right|$.

Proof. Note that under the assumptions above, the norm of the linear operator $\mathcal{S}: \mathcal{H} \rightarrow \mathcal{H}$

$$
(\mathcal{S} \mathbf{y})_{n}(z)=\frac{r y_{n-1}(z)+r y_{n+1}(z)}{(\sqrt{-i} \sqrt{i+i n \omega+z}-1)}
$$

is smaller than 1 , since $|\sqrt{-i} \sqrt{i+i n \omega+z}-1| \geqslant|\sqrt{i+i n \omega+z}|-1 \geqslant \sqrt{|\operatorname{Re}(z)|}-$ $1>2 r$. We then have

$$
\mathbf{y}=\frac{(\mathcal{I}-\mathcal{S})^{-1} \mathbf{g}}{(\sqrt{-i} \sqrt{i+i n \omega+z}-1)}
$$

Proposition 5. For every $r \in \mathbb{C}$, there are at most finitely many $z=z_{1}, \ldots, z_{l_{r}}$ for which the homogeneous equation (10) has a nonzero solution $\mathbf{y}$ in $\mathcal{H}$. For all other $z$, there exists a unique solution to (9). The function $\sqrt{z-z_{0}} \mathbf{y}$ is analytic in both $\sqrt{z-z_{0}}$ and $r$, and it can be analytically continued on the Riemann surface of $\sqrt{i+i n \omega+z}$ to $\arg z \in(-3 \pi / 2,3 \pi / 2)$. (in other words, one can rotate the branch cut in the left half complex plane) Moreover, $z_{1}, \ldots, z_{l_{r}}$ are either poles (in $\sqrt{z-z_{0}}$ ) or removable singularities of $\mathbf{y}$, and $y_{n}\left(n \neq n_{0}\right)$ is analytic in $\sqrt{z-z_{0}}$ when $z$ is close to $z_{0}$.

Proof. We consider the equation

$$
\mathbf{y}^{[m]}=\mathcal{C}_{m} \mathbf{y}^{[m]}+\frac{1}{(\sqrt{-i} \sqrt{i+i n \omega+z}+m \sqrt{i})} \mathbf{f}
$$

Since $\mathcal{C}_{m}$ is compact, analytic in both $r$ and $\sqrt{z-z_{0}}$, and invertible for $|\operatorname{Re}(z)|>$ $(2 r+1)^{2}$, it follows from the analytic Fredholm alternative (see [6] Vol 1, Theorem VI.14, pp. 201) that the proposition is true for every $\mathbf{y}^{[\mathrm{m}]}$ (note that the solution of the inhomogeneous equation exists for $|\operatorname{Re}(z)|>(2 r+1)^{2}$, thus there can only be finitely many isolated singularities). Uniqueness of the solution implies $\mathbf{y}^{[m]}=\mathbf{y}^{[m+1]}$ for all $r \in \mathbb{C}, \operatorname{Re}(z)>-m^{2}$. Thus we naturally define the analytic continuation of the solution to be $\mathbf{y}:=\mathbf{y}^{[\mathrm{m}]}$. Analytic continuation on the Riemann surface follows from the fact that for fixed $r, z$ ( $z$ not on the branch cut) slightly rotating the branch cut does not change the value of $\sqrt{i+i n \omega+z}$ for any $n \in \mathbb{Z}$. Uniqueness of the solution thus ensures $\mathbf{y}$ also remains unchanged.

Assume $y_{n}(z) \sim b_{n}\left(z-z_{0}\right)^{-1 / 2}$ as $z \rightarrow z_{0}$. It is easy to see from (9) that

$$
\begin{aligned}
& (\sqrt{-i} \sqrt{i+i n \omega+z}-1) b_{n}=r b_{n-1}+r b_{n+1}\left(n \neq n_{0}\right) \\
& \left(\sqrt{-i} \sqrt{i+i n_{0} \omega+z_{0}}-1\right) b_{n_{0}}=r b_{n_{0}-1}+r b_{n_{0}+1}-(1 / 2+r) i^{3 / 2} \int_{-\infty}^{\infty} \psi_{0}(x) d x
\end{aligned}
$$

The unique solution of this recurrence relation is obviously

$$
b_{n_{0}}=(1 / 2+r) i^{3 / 2} \int_{-\infty}^{\infty} \psi_{0}(x) d x, b_{n}=0\left(n \neq n_{0}\right)
$$


Corollary 6. For every $r \in \mathbb{C}$, (8) has a unique solution $\tilde{\psi} \cdot \sqrt{p} \tilde{\psi}$ is meromorphic in $p$ with square root branches at every in $\omega(n \in \mathbb{Z})$ and poles at $\left\{p_{k}+\right.$ in $\left.\omega\right\}$ $\left(k=1,2 \ldots l_{r}, n \in \mathbb{Z}\right)$.

Proof. In order to recover $p=i+i n \omega+z$ from the solution to (9), we only need to show $y_{n}(z)=y_{n \mp 1}(z \pm \omega i)$. To this end, note that by (9) we have

$$
\begin{aligned}
(\sqrt{-i} \sqrt{i+i n \omega+z}-1) y_{n \mp 1}(z \pm \omega i) & \\
& =r y_{n \mp 1-1}(z \pm \omega i)+r y_{n \mp 1+1}(z \pm \omega i)+f_{n \mp 1}(z \pm \omega i)
\end{aligned}
$$

which is the same equation as (9) since $f_{n \mp 1}(z \pm \omega i)=f_{n}$.

Thus, uniqueness of the solution (Proposition [5) implies $y_{n}(z)=y_{n \mp 1}(z \pm \omega i)$. Note that we need to choose the same branch for all $\sqrt{i+i n \omega+z}$.

We conclude this section with a few observations about the positions of the poles of $\tilde{\psi}$, including the well-known result of complete ionization (see [5, 7, 16]).

Proposition 7. For $r>0, \mathbf{y}$ has no pole on the imaginary axis or the right half complex plane, with the usual choice of branch.

Proof. In view of Proposition [5] we only need to show the homogeneous equation (10) has no nonzero solution in $\mathcal{H}$. Multiplying (10) by $\overline{y_{n}}(z)$ and summing in $n$ we get

$$
\sum_{n=-\infty}^{\infty}(\sqrt{-i} \sqrt{i+i n \omega+z}-1)\left|y_{n}\right|^{2}=2 r \sum_{n=-\infty}^{\infty} \operatorname{Re}\left(y_{n-1} \overline{y_{n}}\right)
$$

which implies

$$
\sum_{n=-\infty}^{\infty} \sqrt{-i} \sqrt{i+i n \omega+z}\left|y_{n}\right|^{2}
$$

must be real.

If $\operatorname{Re}(z) \geqslant 0$ then $\operatorname{Im}(\sqrt{-i} \sqrt{i+i n \omega+z}) \leqslant 0$ for all $n$ and $\operatorname{Im}(\sqrt{-i} \sqrt{i+i n \omega+z})<$ 0 for all $n<-(1+|z|) / \omega$. Thus $y_{n}=0$ for all $n<-(1+|z|) / \omega$ and (10) implies $\mathbf{y}=0$.

Proposition 8. For $r>0$, y has no pole on the imaginary axis for any choice of branch.

Proof. Similar to the above. Note that $\operatorname{Re}(z)=0$ implies $\operatorname{Im}(\sqrt{-i} \sqrt{i+i n \omega+z})=$ 0 for all $n>-(1+\operatorname{Im}(z)) / \omega$ and $\operatorname{Im}(\sqrt{-i} \sqrt{i+i n \omega+z})$ has the same sign (and nonzero) for all $n<-(1+\operatorname{Im}(z)) / \omega$.

Proposition 9. Solutions of the homogeneous equation (10) exist in negative conjugate pairs, in the sense that if $z_{1}$ is a pole of $\tilde{\psi}$, then $-\overline{z_{1}}$ is also a pole (with a different choice of branch, see proof and comments below).

Proof. Simply note that $(-i)^{1 / 2} \sqrt{i+i n \omega+z}=\overline{(-i)^{1 / 2} \sqrt{i+i n \omega-\bar{z}}}$ if we choose the branches in such a way that in the upper half complex plane the two square roots are the same, while in the lower half plane they are opposite.

In view of the above propositions, we will concentrate our study of resonances on the left half complex plane. The author believes that the imaginary line on the non-principal Riemann surface is a singularity barrier, and the Proposition 9 
provides a pseudo-analytic continuation across the barrier. We will not discuss the details in this paper.

3.3. The homogeneous equation. As we mentioned in the introduction, poles of $\mathbf{y}$ in the left half complex plane correspond to resonances of the system. According to Proposition 5. finding these poles is essentially the same as finding solutions to the homogeneous equation (10) in $\mathcal{H}$.

Lemma 10. Assume the nonzero vector $\mathbf{u}=\left\{u_{n}\right\}$ satisfies the homogeneous recurrence relation (10), and that

$$
\sum_{n=0}^{\infty}\left(1+|n|^{3 / 2}\right)\left|u_{n}\right|^{2}<\infty
$$

Assume also that the nonzero vector $\mathbf{v}=\left\{v_{n}\right\}$ satisfies (10) and

$$
\sum_{n=-\infty}^{0}\left(1+|n|^{3 / 2}\right)\left|v_{n}\right|^{2}<\infty
$$

Then the homogeneous equation (10) has a nonzero solution in $\mathcal{H}$ if and only if the discrete Wronskian $W:=u_{n} v_{n+1}-v_{n} u_{n+1}=0$. The solution, if it exists, is a constant multiple of $\mathbf{u}$ (or equivalently $\mathbf{v}$ ).

Proof. If $r=0$ the lemma is trivial. Assume $r>0$. We first note that the recurrence relation (10) implies

(1) $W$ is independent of $n$.

(2) for any $n$ and any nonzero vector $\mathbf{x}$ satisfying that recurrence relation, we have $\left|x_{n}\right|^{2}+\left|x_{n+1}\right|^{2} \neq 0,\left|x_{n}\right|^{2}+\left|x_{n+2}\right|^{2} \neq 0(n \neq-1)$.

Now assume $W=0$. Since $\mathbf{v}$ is nonzero, there exists $m$ for which $v_{m} \neq 0$. Thus we have $u_{m \pm 1}=\left(u_{m} / v_{m}\right) v_{m \pm 1}$. Since $\mathbf{u} \neq 0$ we must have $u_{m} \neq 0$, for otherwise $u_{m \pm 1}=u_{m}=0$. If $v_{m \pm 1}=0$ then $u_{m \pm 1}=0$, which implies $\left|u_{m}-\left(u_{m} / v_{m}\right) v_{m}\right|^{2}+$ $\left|u_{m \pm 1}-\left(u_{m} / v_{m}\right) v_{m \pm 1}\right|^{2}=0$, meaning $\mathbf{u}=\left(u_{m} / v_{m}\right) \mathbf{v}$. If $v_{m \pm 1} \neq 0$ then $u_{m \pm 1} \neq 0$, which inductively implies again $\mathbf{u}=\left(u_{m} / v_{m}\right) \mathbf{v}$. Therefore $\mathbf{u}$ solves (10) in $\mathcal{H}$.

If $W \neq 0$ then clearly $\mathbf{u}$ and $\mathbf{v}$ are the two linearly independent solutions of the second order difference equation (10). Furthermore, we have $\liminf \operatorname{lin}_{n<0}\left|u_{n}\right|>0$ and $\liminf \operatorname{in}_{n>0}\left|v_{n}\right|>0$, since $\lim \sup _{n>0}\left|u_{n}\right|<$ const. $|n|^{-3 / 4}$ and $\lim \sup _{n<0}\left|v_{n}\right|<$ const. $|n|^{-3 / 4}$ but $u_{n} v_{n+1}-v_{n} u_{n+1}$ is a nonzero constant. Therefore no nonzero linear combination of $\mathbf{u}$ and $\mathbf{v}$ can be in $\mathcal{H}$. Since a second order difference equation cannot have any other solution, there is no nonzero solution of (10) in $\mathcal{H}$.

We now give a constructive description of $\mathbf{u}$ and $\mathbf{v}$. For convenience let $h_{n}(z)=$ $(\sqrt{-i} \sqrt{i+i n \omega+z}-1)$. We choose $n_{1,2} \in \mathbb{Z}$ so that $\left|h_{n}\right|>2|r|$ for all $n \geqslant n_{1}>0$ and $n \leqslant n_{2}<0$. Let $\mathcal{I}$ be the identity operator. We define $\mathcal{H}_{1,2}$ by

$$
\begin{aligned}
\|\mathbf{x}\|_{1}^{2} & =\sum_{n=n_{1}}^{\infty}\left(1+|n|^{3 / 2}\right)\left|x_{n}\right|^{2} \\
\|\mathbf{x}\|_{2}^{2} & =\sum_{n=-\infty}^{n_{2}}\left(1+|n|^{3 / 2}\right)\left|x_{n}\right|^{2}
\end{aligned}
$$


Proposition 11. There exist $\mathbf{u}$ and $\mathbf{v}$, analytic in $r$ and ramified analytic in $z$, satisfying the conditions described in Lemma 10. Moreover, $\mathbf{u}(z \pm \omega i)=$ const.u $(z)$ and $\mathbf{v}(z \pm \omega i)=$ const $\mathbf{v}(z)$.

Proof. Let $\mathcal{T}_{1}: \mathcal{H}_{1} \rightarrow \mathcal{H}_{1}$

$$
\left(\mathcal{T}_{1} y\right)_{n}= \begin{cases}\frac{r}{h_{n}}\left(y_{n-1}+y_{n+1}\right) & n>n_{1} \\ \frac{r}{h_{n}} y_{n+1} & n=n_{1}\end{cases}
$$

Let $\mathbf{a}=\left(r / h_{n_{1}}, 0,0 \ldots\right) \in \mathcal{H}_{1}$.

The equation

$$
\mathbf{u}=\mathcal{T}_{1} \mathbf{u}+\mathbf{a}
$$

has a unique solution

$$
\mathbf{u}=\left(\mathcal{I}-\mathcal{T}_{1}\right)^{-1} \mathbf{a}=\mathbf{a}+\mathcal{T}_{1} \mathbf{a}+\mathcal{T}_{2}^{2} \mathbf{a} \ldots
$$

since clearly $\left\|\mathcal{T}_{1}\right\|<1$. It is easy to see that $\mathbf{u}$ satisfies

Thus the recurrence relation

$$
u_{n}= \begin{cases}\frac{r}{h_{n}}\left(u_{n-1}+u_{n+1}\right) & n>n_{1} \\ \frac{r}{h_{n}}\left(u_{n+1}+1\right) & n=n_{1}\end{cases}
$$

$$
u_{n}=\frac{h_{n}}{r} u_{n+1}-u_{n+2}
$$

extends $\mathbf{u}$ to a solution of the homogeneous equation (10). In particular $u_{n_{1}-1}=1$. This solution $\mathbf{u}$ is analytic in $r$ and $z$ (ramified) locally since $\mathcal{T}_{1}$ and $h_{n}$ are analytic in $r$ and $z$ (ramified), and the uniform limit of analytic functions is analytic. As $r$ or $|\operatorname{Im}(z)|$ increases we may analytically continue $\mathbf{u}$ by considering some $n_{3}>n_{1}$ so that $\left|h_{n}\right|>2|r|$ for all $n \geqslant n_{3}$. Using the same procedure as we did for $n_{1}$ we get $\tilde{\mathbf{u}}$. It is easy to see that $\mathbf{u}=u_{n_{3}} \widetilde{\mathbf{u}}$ for they both satisfy the contractive recurrence relation (in the sup norm)

$$
u_{n}= \begin{cases}\frac{r}{h_{n}}\left(u_{n-1}+u_{n+1}\right) & n>n_{3} \\ \frac{r}{h_{n}}\left(u_{n+1}+u_{n_{3}}\right) & n=n_{3}\end{cases}
$$

Note that this implies $u_{n} \neq 0$ for large $n$.

The analytic continuation of $\mathbf{u}$ is, up to a scalar multiple, periodic in $z$. Note that $\mathbf{u}^{ \pm}(z)=\mathbf{u}(z \pm \omega i)$ satisfies (for large $n_{3}>n_{1}$ )

$$
u_{n}^{ \pm}= \begin{cases}\frac{r}{h_{n \pm 1}}\left(u_{n-1}^{ \pm}+u_{n+1}^{ \pm}\right) & n>n_{1} \\ \frac{r}{h_{n \pm 1}}\left(u_{n+1}^{ \pm}+u_{n_{3}}^{ \pm}\right) & n=n_{3}\end{cases}
$$


while $\mathbf{u}$ satisfies

$$
u_{n \pm 1}= \begin{cases}\frac{r}{h_{n \pm 1}}\left(u_{n \pm 1-1}+u_{n \pm 1+1}\right) & n>n_{3} \\ \frac{r}{h_{n \pm 1}}\left(u_{n \pm 1+1}+u_{n_{3} \pm 1}\right) & n=n_{3}\end{cases}
$$

Thus $\mathbf{u}(z \pm \omega i)=\frac{u_{n_{3}}(z \pm \omega i)}{u_{n_{3} \pm 1}(z)} \mathbf{u}(z)$.

The construction of $\mathbf{v}$ is very similar, namely $\mathbf{v}=\left(\mathcal{I}-\mathcal{T}_{2}\right)^{-1} \mathbf{b}$ where $\mathcal{T}_{2}: \mathcal{H}_{2} \rightarrow$ $\mathcal{H}_{2}$

$$
\left(\mathcal{T}_{2} y\right)_{n} \rightarrow \begin{cases}\frac{r}{h_{n}}\left(y_{n-1}+y_{n+1}\right) & n<n_{2} \\ \frac{r}{h_{n}} y_{n-1} & n=n_{2}\end{cases}
$$

and $\mathbf{b}=\left(\ldots, 0,0, r / h_{n_{2}}\right)$.

Proposition 12. $W$ is analytic in $r$ and ramified analytic in $z$. Moreover, $W(z)=$ 0 if and only if $W(z \pm \omega i)=0$.

Proof. The first part is obvious. The second part follows from the relation $\mathbf{u}(z \pm$ $\omega i)=\frac{u_{n_{3}}(z \pm \omega i)}{u_{n_{3} \pm 1}(z)} \mathbf{u}(z)$ (see the proof of the previous proposition) and the fact that $u_{n_{3}} \neq 0$.

Remark 2. Another way of constructing $\mathbf{u}$ and $\mathbf{v}$ is by using continued fractions, see [5]. The continued fraction expression is slightly simpler in this particular case, but our iteration method can be easily generalized to trigonometric polynomial potentials mentioned in section 2 .

3.4. Resonance for small $\mathbf{r}$. We assume $r>0$ and analyze the resonances of the system for small $r$ (relative to $\omega$ ) by locating zeros of $W$, in view of Lemma 10 , Since we will need to consider different branch choices, we write for convenience $h_{n}(z)=\left((-i)^{1 / 2} \sqrt{i+i n \omega+z}-1\right)$ where the power $1 / 2$ always indicates the usual choice of branch.

Lemma 13. For every choice of branch, there exists a constant $c$ so that when $\omega>c\left(r+r^{2}\right)$, we have $\left|h_{n}\right|>2 r$ for all $n \neq 0$.

Proof. Recall that for a branch cut at $\left(e^{i \theta} \infty, z_{0}\right)(\cos \theta \neq 0)$, we consider the stripshaped region $\Omega_{b}:=\left\{|\operatorname{Im}(z)-\rho \sin \theta|<\frac{4}{5} \omega, \operatorname{Re}(\mathrm{z})=\rho \cos \theta, \rho \in \mathbb{R}\right\}$. It is easy to see that $c_{1}:=\inf _{n \neq 0, z \in \Omega_{b}}\left|\frac{z}{\omega}-i n\right|>0$. Therefore $\left|h_{n}(z)\right|=\left|(-i)^{1 / 2} \sqrt{i+i n \omega+z}-1\right|=$ $\frac{|i n \omega+z|}{|\sqrt{i+i n \omega+z}+\sqrt{i}|} \geqslant \frac{|i n \omega+z|}{\sqrt{|i n \omega+z|}+2} \geqslant \frac{c_{1} \omega}{\sqrt{c_{1} \omega}+2}>2 r$ if $\sqrt{c_{1} \omega}>2 r+2 \sqrt{r}$. Note that $\frac{x^{2}}{x+2}$ is an increasing function for $x>0$.

Proposition 14. For small $r$, there is a unique nonzero solution of the homogeneous equation (10) in the left half complex plane with the usual choice of branch. 
Moreover, the solution satisfies

$$
z=\left(\frac{2 i}{(1+\omega)^{1 / 2}-1}-\frac{2 i}{i^{-1 / 2} \sqrt{(1-\omega) i}-1}+\sigma(r)\right) r^{2}
$$

where $\sigma(r)$ is analytic in $r$ and $\sigma(0)=0$.

Proof. We choose $n_{1}=1, n_{2}=-1$ to construct $\mathbf{u}$ and $\mathbf{v}$. Thus $u_{0}=v_{0}=1$ and $W=v_{1}-u_{1}$. We calculate by iterations

$$
\begin{gathered}
u_{1}=\frac{r}{h_{1}}+\frac{r^{3}}{h_{1}^{2} h_{2}}+\frac{r^{5}}{h_{1}^{5}} R_{1} \\
v_{1}=\frac{h_{0}}{r}-v_{-1}=\frac{h_{0}}{r}-\frac{r}{h_{-1}}-\frac{r^{3}}{h_{-1}^{2} h_{-2}}-\frac{r^{5}}{h_{1}^{5}} R_{2} \\
W=\frac{h_{0}}{r}-\frac{r}{h_{-1}}-\frac{r}{h_{1}}-\frac{r^{3}}{h_{1}^{2} h_{2}}-\frac{r^{3}}{h_{-1}^{2} h_{-2}}-\frac{r^{5}}{h_{1}^{5}} R_{1}-\frac{r^{5}}{h_{1}^{5}} R_{2}
\end{gathered}
$$

where $R_{1,2}$ are bounded for $\omega>c\left(r+r^{2}\right)$. Note that $\left|h_{0}(z)\right|=\left|\sqrt{i+z}-i^{1 / 2}\right| \geqslant|z| / 2$ and $\left|\frac{r}{h_{n}}\right| \leqslant \frac{\sqrt{c_{1} \omega}+2}{c_{1} \omega} r$ for all $n \neq 0$.

Now, if $\omega$ is fixed and $r$ is small, $W=0$ implies $h_{0}(z)=O\left(r^{2}\right)$. Hence we must have $z=O\left(r^{2}\right)$. In addition, we need to make the choice of branch so that $\sqrt{i}$ is in the first quadrant. Thus we let $z=\left(a_{0}+\sigma\right) r^{2}$ where $\sigma=o(1)$, and we see that

$$
\frac{W}{r}=\left(\frac{a_{0}}{2 i}-\frac{1}{(1+\omega)^{1 / 2}-1}-\frac{i^{1 / 2}}{\sqrt{(1-\omega) i}-i^{1 / 2}}\right)(1+o(1))
$$

Thus we have

$$
a_{0}=\frac{2 i}{(1+\omega)^{1 / 2}-1}-\frac{2 i}{i^{-1 / 2} \sqrt{(1-\omega) i}-1}
$$

For small $r, W$ is clearly analytic in both $r$ and $\sigma$. Since the value of $W$ depends only on $\bigcup_{n}\left\{z:|z-i n \omega|<2 a_{0} r^{2}\right\}$, there are exactly two different $W$ with different choices of branch, namely $W_{1}: \operatorname{Re}(\sqrt{i})>0, \operatorname{Re}(\sqrt{-i})>0$ and $W_{2}: \operatorname{Re}(\sqrt{i})>$ $0, \operatorname{Re}(\sqrt{-i})<0$. However, according to Proposition 8 and Proposition 9, they are in fact negative conjugates to each other, and only one will be in the left half complex plane. We thus take $W=W_{1}$ for its branch is consistent with the usual branch.

It is easy to verify that

$$
\begin{gathered}
\left.\frac{W}{r}\right|_{r=0, \sigma=0}=0 \\
\left.\frac{\partial}{\partial \sigma}\left(\frac{W}{r}\right)\right|_{r=0, \sigma=0}=-\frac{i}{2} \neq 0
\end{gathered}
$$

Therefore it follows from the implicit function theorem that the position of the zero of $W$ is given by

$$
z=\left(\frac{2 i}{(1+\omega)^{1 / 2}-1}-\frac{2 i}{i^{-1 / 2} \sqrt{(1-\omega) i}-1}+\sigma(r)\right) r^{2}
$$

where $\sigma(r)$ is analytic in $r$ and $\sigma(0)=0$. 
$\sigma(r)$ can be found asymptotically by iterating $\sigma(r)-\frac{2 i W}{r}$ as in the standard proof of the implicit function theorem.

Since the usual choice of branch is consistent with $W$, the zero of $W$ is visible.

Corollary 15. For $r$ small and $\omega>1$, the position of the resonance satisfies $\lambda_{1} \sim-\frac{2 \sqrt{\omega-1}}{\omega} r^{2}-\frac{2 \sqrt{\omega+1}}{\omega} r^{2} i$.

Proof. The corollary follows from the expression of $a_{0}$ with the usual choice of branch. The fact that it is indeed a resonance, i.e. a pole of $\mathbf{y}$, will be established in the next subsection.

Remark 3. In the case $\omega \gg 1+r^{2}$, an analogous analysis shows that the position of the resonance is given by $\lambda_{1} \sim-\frac{2 r^{2}}{\sqrt{\omega}}-\frac{2 r^{2} i}{\sqrt{\omega}}$.

Proposition 16. For small $r$ the poles (in one vertical array) of $\tilde{\psi}$ are simple and the residues are nonzero for generic $\mathbf{f}$.

Proof. We note that the order of the pole of $\left(\mathcal{I}-\mathcal{C}_{m}\right)^{-1}$ equals the order of the corresponding zero of $\mathcal{I}-\mathcal{C}_{m}$, which is a constant by the argument principle (see Lemma 18 below), since $\mathcal{I}-\mathcal{C}_{m}$ is analytic in $z$. It is easy to verify that when $r=0$ the zero of $\mathcal{I}-\mathcal{C}_{m}$ is of order one. Thus the poles are simple.

Let $z=G(r)$ be the continuous functions satisfying $W(G(r), r)=0, G(0)=0$. We consider the residue

$$
P(r)=\frac{1}{2 \pi i} \oint_{|\zeta-G(r)|=\epsilon} y_{0}(\zeta, r) d \zeta
$$

Obviously $P(r)$ is analytic in $r$. For generic $\mathbf{f}, P(0) \neq 0$ (in which case $\mathbf{y}$ can be found explicitly). Thus $P(r) \neq 0$ for small $r$.

3.5. Resonances in general. Having analyzed the zeros of $W$ for small $r$, we proceed to consider the case for general $r$, as well as the poles of $\mathbf{y}$.

For convenience we study the region $\Omega_{\theta, \epsilon}:=\left\{z: \operatorname{Im}(z) \in\left[\rho \sin \theta+\frac{z_{0}}{2}-\frac{1}{2} \omega+\right.\right.$ $\left.\left.\epsilon, \rho \sin \theta+\frac{z_{0}}{2}+\frac{1}{2} \omega+\epsilon\right), \operatorname{Re}(\mathrm{z})=\rho \cos \theta, \rho \in \mathbb{R}\right\} \bigcap\left\{z:|\operatorname{Re}(z)|<(2|r|+2)^{2}\right\}$, the branch cut being placed at $\left(e^{i \theta} \infty, z_{0}\right)(\cos \theta \neq 0)$. It is easy to see that there is exactly one zero and one branch point inside this region for small $r$ (cf. Section 3.4). We note that as long as $z$ is not located on a branch cut, we may rotate the cut slightly without changing $W$.

Lemma 17. For every $r, W$ has finitely many zeros in $\bigcup_{|\cos \theta|>c_{b}>0} \Omega_{\theta, \epsilon}$ where $c_{b}$ is arbitrary.

Proof. By Lemma 4, there is no zero for $|\operatorname{Re}(z)|>(2|r|+1)^{2}$ and the zeros are isolated. Since the Riemann surface of the square root has only two sheets and the region $\Omega_{\theta, \epsilon}$ is bounded, $W$ can only have finitely many zeros.

Lemma 18. Assume for some $r_{0}$ and arbitrarily small $\epsilon>0$, with the branch choice $\arg (z) \in(\theta-\epsilon, \theta+2 \pi+\epsilon)(-2 \pi<\theta \leqslant 2 \pi, \cos \theta \neq 0), W$ has finitely many zeros in $\Omega_{\theta, \epsilon}$. Then the number of zeros remains a constant if $r$ is close to $r_{0}$. Furthermore, each zero moves continuously with respect to $r$.

Proof. The lemma follows from standard complex analysis arguments. Suppose $W\left(z, r_{0}\right)$ has zeros $z_{1}, z_{2}, \ldots z_{m}$ inside $\Omega_{0}$ and $\tilde{z}_{m+1}, \ldots \tilde{z}_{m+l}$ on $\partial \Omega_{0}$. Since $z \in \Omega_{\theta, \epsilon}-$ $\Omega_{\theta, 0}$ iff $z-i \omega \in \Omega_{\theta, 0}-\Omega_{\theta, \epsilon}$, we let $z_{m+k}=\tilde{z}_{m+k}+i \omega(1 \leqslant k \leqslant l)$. We may 
choose small $\epsilon>0$ so that $W\left(z, r_{0}\right)$ has zeros $z_{1,} z_{2, \ldots} z_{m+l}$ in $\Omega_{\theta, \epsilon}$ for $\arg (z) \in$ $(\theta-\epsilon, \theta+2 \pi+\epsilon)$, and no other zero in $\Omega_{\theta, 2 \epsilon}$ for $\arg (z) \in(\theta-2 \epsilon, \theta+2 \pi+2 \epsilon)$. Let $0<\delta<\epsilon$ be small so that there is at most one zero or branch point inside any circle of radius $2 \delta$, and $W\left(z, r_{0}\right)$ is analytic (with a suitable choice of branch) in $\left|z-z_{n}\right|<2 \delta$. Since $W$ is analytic in both $z$ and $r$, it follows from the argument principle that for $r$ very close to $r_{0}$

$$
M_{n}(r)=\frac{1}{2 \pi i} \oint_{\left|\zeta-z_{n}\right|=\delta} \frac{\frac{\partial}{\partial \zeta} W(\zeta, r)}{W(\zeta, r)} d \zeta=1
$$

Now we consider the compact region $\Omega^{\prime}:=\{z: \arg (z) \in[\theta-\epsilon, \theta+2 \pi+\epsilon]\} \bigcap \overline{\Omega_{\theta, \epsilon}} \backslash$ $\bigcup_{n=1}^{m}\left\{z:\left|z-z_{n}\right|<\delta\right\}$. Clearly $\left|W\left(z, r_{0}\right)\right|>0$ for all $z \in \Omega^{\prime}$. Since $W$ is jointly uniformly continuous in $z$ and $r$, we have $|W(z, r)|>0$ for all $z \in \Omega^{\prime}, r$ close to $r_{0}$.

Thus the number of zeros is locally a constant and they move continuously with respect to $r$.

Proposition 19. For every $r$ there are finitely many zeros of $W$ in any strip $\{z: \operatorname{Im}(z) \in[\tilde{z}, \tilde{z}+\omega), \operatorname{Re}(z) \in \mathbb{R}\}$ for all choices of branch within $|\cos \theta|>c_{b}>0$, and the position of each zero changes continuously with respect to $r$.

Proof. The conclusion follows from Proposition [12. Lemma 17 and 18. Note that we may choose $\theta$ arbitrarily, thus covering the whole Riemann surface (except for the imaginary lines).

As we have shown in Proposition [5] and Lemma [10, all poles of $\mathbf{y}$ are located where $W=0$. We summarize the results as

Proposition 20. For generic $r$ and $\mathbf{f}, \mathbf{y}(z, r)$ has finitely many arrays of poles for any choice of branch with $|\cos \theta|>c_{b}>0$. Their residues $A_{k, n}$ satisfy the recurrence relation

$$
\left((-i)^{1 / 2} \sqrt{i+n \omega i-\lambda_{1}}-1\right) A_{k, n}=r A_{k, n-1}+r A_{k, n+1}
$$

and $\mathbf{A}_{k} \in \mathcal{H}$.

Proof. The first part is simply a rephrasing of previous results (cf. Proposition 19). The recurrence relation for residues follows from the fact that

$$
A_{k, n}=\frac{1}{2 \pi i} \oint_{|\zeta-G(r)|=\epsilon} y_{k, n}(\zeta, r) d \zeta
$$

satisfies the homogeneous equation (10) since $\mathbf{y}$ satisfies (9) and

$$
\oint_{|\zeta-G(r)|=\epsilon} f_{n}(\zeta, r) d \zeta=0
$$

The above expression for $P_{n}$ also implies $\mathbf{A}_{k} \in \mathcal{H}$ since, by Hölder's inequality

$$
\begin{aligned}
& \sum_{n=-\infty}^{\infty}\left(1+|n|^{3 / 2}\right)\left|A_{k, n}\right|^{2} \leqslant \sum_{n=-\infty}^{\infty}\left(1+|n|^{3 / 2}\right) \oint_{|\zeta-G(r)|=\epsilon}\left|y_{n}(\zeta, r)\right|^{2} d|\zeta| \\
& \quad=\oint_{|\zeta-G(r)|=\epsilon} \sum_{n=-\infty}^{\infty}\left(1+|n|^{3 / 2}\right)\left|y_{n}(\zeta, r)\right|^{2} d|\zeta| \leqslant\left.\sup _{\left|\zeta-G_{1}(r)\right|=\epsilon}|| \mathbf{y}(\zeta, r)\right|^{2}<\infty
\end{aligned}
$$


the last inequality following from the continuity of $\mathbf{y}$ (see also Section 3.6 below).

3.6. Proof of Theorem 1. As we have mentioned before, we will take the inverse Laplace transform of $\hat{\psi}$ and deform the contour, collecting contributions from the poles in the process. We first provide the necessary estimates.

Lemma 21. Assume $\operatorname{supp} \psi_{0} \in[-M, M]$, then $\sqrt{p} f(x, p)$, where $f(x, p)$ is as defined in Section Q 2 is analytic in $\sqrt{p}$ with a square root branch at zero. Moreover,

$$
f(x, p)=\frac{\psi_{0}(x)}{p}+O\left(p^{-3 / 2}\right)+O\left(p^{-3 / 2} e^{M i^{3 / 2} \sqrt{p}}\right)
$$

for large $|p|$.

Proof. By integration by parts we have

$$
\begin{gathered}
f(x, p)=\frac{\psi_{0}(x)}{2 p}-\frac{e^{-i^{3 / 2} \sqrt{p} x}}{2 p} \int_{+\infty}^{x} e^{i^{3 / 2} \sqrt{p} s} \psi_{0}^{\prime}(s) d s \\
+\frac{\psi_{0}(x)}{2 p}-\frac{e^{i^{3 / 2}} \sqrt{p} x}{2 p} \int_{-\infty}^{x} e^{-i^{3 / 2} \sqrt{p} s} \psi_{0}^{\prime}(s) d s \\
=\frac{\psi_{0}(x)}{p}-\frac{\psi_{0}^{\prime}(x)}{2 i^{3 / 2} p^{3 / 2}}+\frac{e^{-i^{3 / 2} \sqrt{p} x}}{2 i^{3 / 2} p^{3 / 2}} \int_{+\infty}^{x} e^{i^{3 / 2} \sqrt{p} s} \psi_{0}^{\prime \prime}(s) d s \\
+\frac{\psi_{0}^{\prime}(x)}{2 i^{3 / 2} p^{3 / 2}}-\frac{e^{i^{3 / 2}} \sqrt{p} x}{2 i^{3 / 2} p^{3 / 2}} \int_{-\infty}^{x} e^{-i^{3 / 2} \sqrt{p} s} \psi_{0}^{\prime \prime}(s) d s \\
=\frac{\psi_{0}(x)}{p}+\frac{i^{-3 / 2}}{2 p^{3 / 2}} \int_{+\infty}^{0} e^{i^{3 / 2} \sqrt{p} u} \psi_{0}^{\prime \prime}(u+x) d u-\frac{i^{-3 / 2}}{2 p^{3 / 2}} \int_{-\infty}^{0} e^{-i^{3 / 2} \sqrt{p} u} \psi_{0}^{\prime \prime}(u+x) d u
\end{gathered}
$$

The lemma then follows.

Lemma 22. $\tilde{\psi}(p)$ satisfies

(1) For any compact region $\Omega_{1} \in \mathbb{C}$ which does not contain any pole of $\tilde{\psi}(p)$, we have

$$
\sup _{p \in \Omega_{1}} \sum_{n=-\infty}^{\infty}\left(1+|n|^{3 / 2}\right)|\tilde{\psi}(p+n \omega i)|^{2}<\infty
$$

In particular,

$$
\sup _{p \in \Omega_{1}} \sum_{n=-\infty}^{\infty}|\tilde{\psi}(p+n \omega i)|<\infty
$$

(2) For any $c \geqslant 0, \int_{c-i \infty}^{c+i \infty}|\tilde{\psi}(p)| d p<\infty$.

(3) For $|\operatorname{Re}(p)|>(2 r+1)^{2}$ we have

$$
\tilde{\psi}(p)=p^{-1 / 2} O(f(0, p))=O\left(p^{-3 / 2}\right)+O\left(p^{-2} e^{M i^{3 / 2} \sqrt{p}}\right)
$$

Note that the $p^{-1 / 2}$ behavior of $\tilde{\psi}(p)$ near the origin does not affect the nature of these estimates, so we omit further discussions of that special case. 
Proof. (1) Recall that $\tilde{\psi}(i+n \omega i+z)=y_{n}(z)$ and that $\mathbf{y} \in \mathcal{H}$, i.e.

$$
\|\mathbf{y}\|^{2}=\sum_{n=-\infty}^{\infty}\left(1+|n|^{3 / 2}\right)\left|y_{n}\right|^{2}<\infty
$$

Since $\mathbf{y}$ is continuous in $z$ on the Riemann surface of the square root, so is $\|\mathbf{y}\|$. Compactness of $\Omega_{1}$ then implies $\sup _{p \in \Omega_{1}}\|\mathbf{y}\|<\infty$, from which the first part follows. The second part follows from the Cauchy-Schwarz inequality

$$
\begin{gathered}
\sum_{n=-\infty}^{\infty} \sup _{p \in \Omega_{1}}|\tilde{\psi}(p+n \omega i)|=\sum_{n=-\infty}^{\infty}\left(1+|n|^{3 / 2}\right)^{-1 / 2}\left(1+|n|^{3 / 2}\right)^{1 / 2} \sup _{p \in \Omega_{1}}|\tilde{\psi}(p+n \omega i)| \\
\leqslant \sum_{n=-\infty}^{\infty}\left(1+|n|^{3 / 2}\right)^{-1} \sum_{n=-\infty}^{\infty}\left(1+|n|^{3 / 2}\right) \sup _{p \in \Omega_{1}}|\tilde{\psi}(p+n \omega i)|^{2}<\infty
\end{gathered}
$$

(2) Note that by Fubini's theorem and Cauchy-Schwarz inequality (cf. part (1)) we have

$$
\begin{gathered}
\int_{c-i \infty}^{c+i \infty}|\tilde{\psi}(p)| d p=\sum_{n=-\infty}^{\infty} \int_{0}^{1}|\tilde{\psi}(c+n \omega i+s i)| d s \\
=\int_{0}^{1} \sum_{n=-\infty}^{\infty}|\tilde{\psi}(c+n \omega i+s i)| d s \\
\leqslant \sup _{p \in[c-s i, c+s i]} \sum_{n=-\infty}^{\infty}|\tilde{\psi}(p+n \omega i)|<\infty
\end{gathered}
$$

(3) The conclusion follows from Lemma 4 and Lemma 21.

Proposition 23. $\psi(x, t)=\frac{1}{2 \pi i} \int_{C_{1}} e^{i^{3 / 2} \sqrt{p}|x|+p t} \tilde{\psi}(p) d p+\frac{1}{2 \pi i} \int_{C_{2}} e^{p t} f(x, p) d p$, where the contours $C_{1,2}$ are as shown in Figure 1] and [2. In the process of deforming the first contour, we collect contributions from the poles and we slightly rotate the branch cut by a small angle $\theta$ if a pole sits on the usual branch cut.

Proof. We first note that

$$
\sup _{\operatorname{Im}(p) \geqslant 0}\left|e^{i^{3 / 2} \sqrt{p}|x|}\right|=1
$$

and

$$
\sup _{\operatorname{Im}(p)<0, s \in \mathbb{R}}\left|e^{i^{3 / 2} \sqrt{p+i s}|x|}\right| \leqslant \sup _{v \in \mathbb{R}}\left|e^{i^{3 / 2} \sqrt{-\operatorname{Im}(p)(-1+i v)}|x|}\right|=e^{c_{1}|x| \sqrt{-\operatorname{Im}(p)}}
$$

where $c_{1}=\sup _{v \in \mathbb{R}} \operatorname{Re}\left(i^{3 / 2} \sqrt{(-1+i v)}\right)<\infty$.

Now, by the Bromwich integral formula

$$
\begin{aligned}
\psi(x, t)=\frac{1}{2 \pi i} \int_{c-i \infty}^{c+i \infty} & e^{p t} \hat{\psi}(x, p) d p \\
= & \frac{1}{2 \pi i} \int_{c-i \infty}^{c+i \infty} e^{i^{3 / 2} \sqrt{p}|x|+p t} \tilde{\psi}(p) d p+\frac{1}{2 \pi i} \int_{c-i \infty}^{c+i \infty} e^{p t} f(x, p) d p
\end{aligned}
$$


By Lemma 21 we have

$$
\begin{aligned}
& \frac{1}{2 \pi i} \int_{c-i \infty}^{c+i \infty} e^{p t} f(x, p) d p \\
&= \frac{\psi_{0}(x)}{2 \pi i} \int_{c-i \infty}^{c+i \infty} \frac{e^{p t}}{p} d p+\frac{1}{2 \pi i} \int_{c-i \infty}^{c+i \infty} e^{p t}\left(f(x, p)-\frac{\psi_{0}(x)}{p}\right) d p \\
& \quad=\psi_{0}(x)+\frac{1}{2 \pi i} \int_{C_{2}} e^{p t}\left(f(x, p)-\frac{\psi_{0}(x)}{p}\right) d p=\frac{1}{2 \pi i} \int_{C_{2}} e^{p t} f(x, p) d p
\end{aligned}
$$

As for the first contour, we only need to show that (along both sides of the branch cuts)

$$
\begin{gathered}
\sum_{n=-\infty}^{\infty} \int_{0}^{-q e^{i \theta}} e^{i^{3 / 2} \sqrt{s+n \omega i}|x|+s t+n \omega i t} \tilde{\psi}(s+n \omega i) d s<\infty \\
\sum_{n=-\infty}^{\infty} \int_{-q e^{i \theta}+n \omega i}^{-q e^{i \theta}+(n+1) \omega i} e^{i^{3 / 2} \sqrt{p}|x|+p t} \tilde{\psi}(p) d p<\infty
\end{gathered}
$$

and if the resonance is visible with the usual (or slightly rotated) branch cut, then

$$
\sum_{n=-\infty}^{\infty}\left|A_{k, n}\right|<\infty
$$

The first two estimates follow from Lemma 22, since

$$
\begin{aligned}
& \left|\sum_{n=-\infty}^{\infty} \int_{0}^{-q e^{i \theta}} e^{i^{3 / 2} \sqrt{s+n \omega i}|x|+s t+n \omega i t} \tilde{\psi}(s+n \omega i) d s\right| \\
& \leqslant\left(\sup _{p \in\left[0,-q e^{i \theta}\right]} \sum_{n=-\infty}^{\infty}|\tilde{\psi}(p+n \omega i)|\right) \int_{0}^{-q e^{i \theta}} e^{c_{1}|x| \sqrt{|s|}+s t} d s<\infty
\end{aligned}
$$

and

$$
\begin{aligned}
& \left|\sum_{n=-\infty}^{\infty} \int_{-q e^{i \theta}+n \omega i}^{-q e^{i \theta}+(n+1) \omega i} e^{i^{3 / 2} \sqrt{p}|x|+p t} \tilde{\psi}(p) d p\right| \\
& \leqslant \sum_{n=-\infty}^{\infty} \int_{0}^{\omega i}\left|e^{i^{3 / 2} \sqrt{-q e^{i \theta}+n \omega i+s}|x|-q e^{i \theta}} t \tilde{\psi}\left(-q e^{i \theta}+n \omega i+s\right)\right| d s \\
& \leqslant e^{c_{1}|x| \sqrt{|q|}-q \cos \theta t}\left(\sup _{p \in[0, \omega i]} \sum_{n=-\infty}^{\infty}|\tilde{\psi}(p+n \omega i)|\right)<\infty
\end{aligned}
$$

The estimates for the resonances follows from proposition 20 and the CauchySchwarz inequality. Since $\mathbf{A}_{k} \in \mathcal{H}$, we have

$$
\begin{aligned}
& \sum_{n=-\infty}^{\infty}\left|A_{k, n}\right|=\sum_{n=-\infty}^{\infty}\left(1+|n|^{3 / 2}\right)^{-1 / 2}\left(1+|n|^{3 / 2}\right)^{1 / 2}\left|A_{k, n}\right| \\
& \leqslant \sum_{n=-\infty}^{\infty}\left(1+|n|^{3 / 2}\right)^{-1} \sum_{n=-\infty}^{\infty}\left(1+|n|^{3 / 2}\right)\left|A_{k, n}\right|^{2}<\infty
\end{aligned}
$$




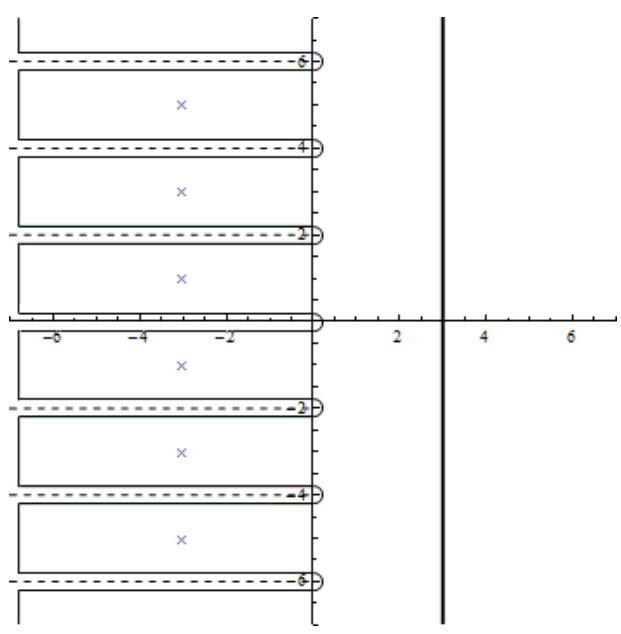

Figure 1. Contour $C_{1}$

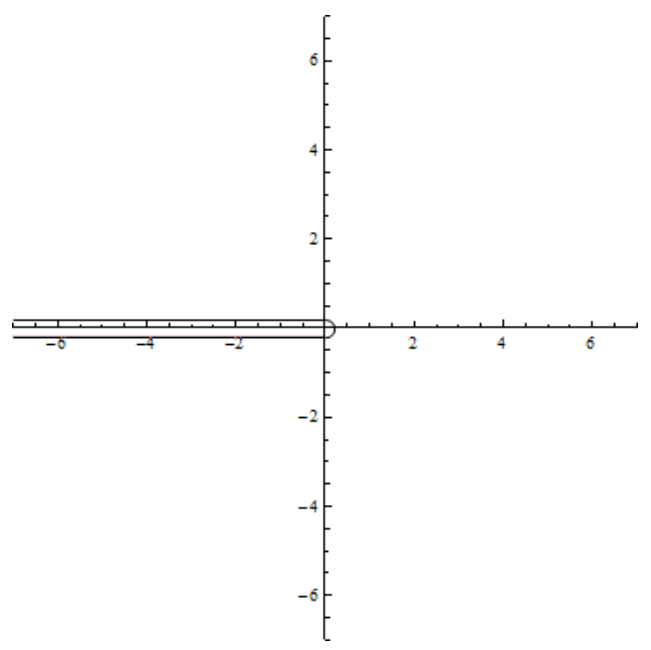

Figure 2. Contour $C_{2}$

Corollary 24. For $t>0$, we may further deform the contour $C_{1}$ to $C_{3}$ by pushing the vertical lines left to infinity.

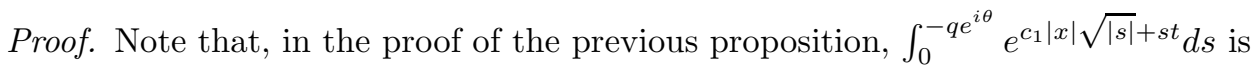
bounded in $\operatorname{Re}(q)>0$ and $e^{c_{1}|x| \sqrt{|q|}-q \cos \theta t} \rightarrow 0$ as $\operatorname{Re}(q) \rightarrow \infty$.

Thus we conclude the proof of Theorem 1 by taking the differences between the upper and lower branches to deform the contour integrals into line integrals. To be exact, if we denote $F_{s}(x, \sqrt{p})=f(x, p), \tilde{\varphi}_{n}(\sqrt{p-n \omega i})=\hat{\psi}(0, p-n \omega i)$, then we take $F(x, p)=F_{s}(x, \sqrt{p})-F_{s}(x,-\sqrt{p})$ and $\varphi(p)=\hat{\psi}_{n}(x, \sqrt{p})-\hat{\psi}_{n}(x, \sqrt{p})$. 
The last part the theorem follows immediately from Watson's Lemma, since $F$ and $\varphi$ are clearly analytic in $\sqrt{p}$ and has sub-exponential growth as $\operatorname{Im}(p) \rightarrow-\infty$ (see Lemma 21 and 221). Note also that $\tilde{\psi}(p) \sim-(1+2 r) f(0, p)$ as $p \rightarrow 0$.

Corollary 1 follows from a direct calculation using (11) and (2).

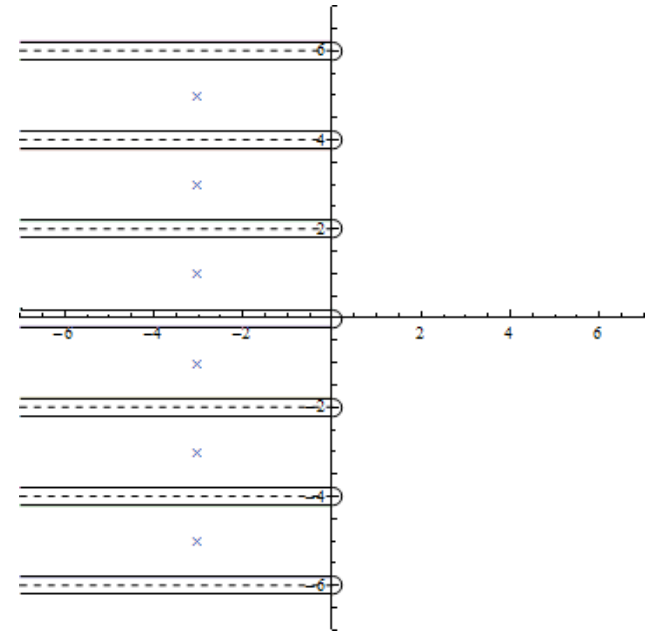

Figure 3. Contour $C_{3}$

\section{Further Discussion and Numerical Results}

In this section we study the physical meaning of the resonances, calculate the positions of the resonances numerically, and discuss the delta potential barrier.

4.1. Metastable states and multiphoton ionization. When a resonance is close to but not on the imaginary axis, it corresponds to a metastable state of the wave function (see [15]). If $|x|$ is not too large, for a moderately long time the wave function is governed by the Gamow vector terms whose resonances are closest to the imaginary axis. Thus, for a fixed initial wave function, the real part of these resonances approximately measure the rate of ionization, that is, the integral of $|\psi|^{2}$ over a fixed spacial interval as a function of $t$.

It has been observed (see [5]) that the rate of ionization changes rapidly when $\omega$ is approximately equal to an integer fraction of the bound state energy (in our case, $\omega=1 / m, m \in \mathbb{N}$ ). This phenomenon is related to multiphoton ionization (see [5, 11, 12, 13, and the references therein), a process in which an electron escapes from the nucleus by absorbing multiple photons at the same time. Since, as we mentioned in the last paragraph, the ionization rate can be measured by the position of resonances, we expect a rapid change in the real part of the resonance $\lambda_{1}$ when $\omega$ is near $1 / m$ and $r$ is small.

Proposition 25. For $\frac{1}{m+1}<\omega \leqslant \frac{1}{m}$, the real part of the resonance is of order $r^{2 m+2}$ for small $r$. 


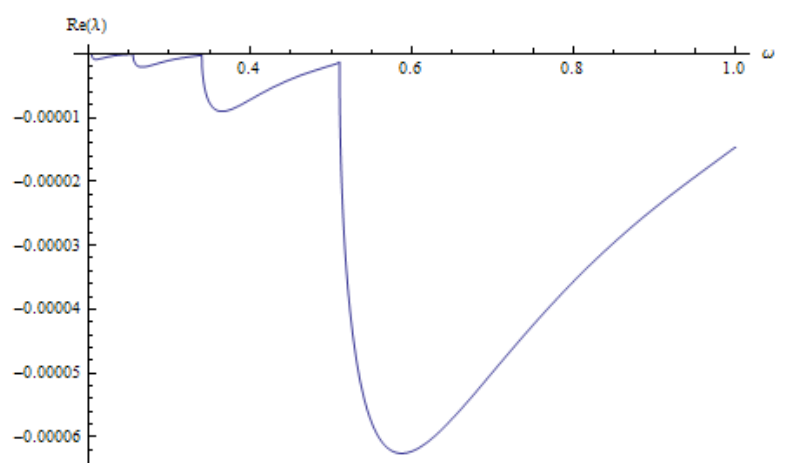

Figure 4. Real part of the resonance as a function of $\omega$

Sketch of Proof: Recalling Proposition 14, we have $z \sim \frac{2 i}{(1+\omega)^{1 / 2}-1}+\frac{2 i}{1-(1-\omega)^{1 / 2}}$. It is easy to see that $\operatorname{Im}\left(h_{n}\right)=O\left(r^{2}\right)$ for $n \geqslant-1 / \omega$.

It can be shown by induction that $\left(\mathcal{T}_{2}^{k} \mathbf{v}\right)_{1}$ is a function of $h_{-1}, h_{-2} \ldots h_{-\left[\frac{k}{2}\right]-1}$ and of order $r^{k+1}$. Moreover, $\left(\mathcal{T}_{2}^{2 k+1} \mathbf{v}\right)_{1}=0$ and $\left(\mathcal{T}_{2}^{2 k} \mathbf{v}\right)_{1} \neq 0$.

Therefore, with the notation $z=\left(\frac{2 i}{(1+\omega)^{1 / 2}-1}+\frac{2 i}{1-(1-\omega)^{1 / 2}}+\sigma\right) r^{2}$, we have $\operatorname{Im}(W)=-\frac{r}{2} \operatorname{Re}(\sigma)(1+o(1))-c_{r} r^{2 m+1}(1+O(r))$. Thus we must have $\operatorname{Re}(\sigma) \sim$ const. $r^{2 m}$.

The above proposition implies that there is indeed a rapid change in the real part of the resonance. Here we confirm this result with numerical calculations (see Figure 4 below) and omit further details of the proof.

4.2. Position of resonance: numerical results. As we have shown in Section 3.4. for small $r$ there is only one resonance in the left half complex plane, for all choices of branch. This is, however, not always the case for general $r$.

We demonstrate the position of resonances in the left half plane by numerically calculating zeros of $W$ for different $r$. In the graph below we show zeros of $W$ plotted with different $r$ and choices of branch, with $\omega=2$.

Based on these numerical results, we make the following observations:

(1) For some values of $r$, such as those between 0.69 and 1.31, there is no visible resonance with the usual choice of branch. In other words, the Gamow vector term in Theorem 1 is absent.

(2) New resonances ("+" marks) emerge as $r$ becomes larger. They can only be "born" from the imaginary axis, according to Proposition 19.

(3) With any given $r$, there does not seem to be more than one resonance visible with the usual choice of branch.

(4) Resonances always move upward with increasing $r$.

(5) New resonances move farther away from the imaginary axis compared to older ones.

(6) "Old" resonances (" $\times "$ marks) do not move arbitrarily close to the imaginary axis with increasing $r$.

4.3. Delta potential barrier. Finally, we briefly discuss the case for the delta potential barrier. The corresponding recurrence relation (see (9)) is 


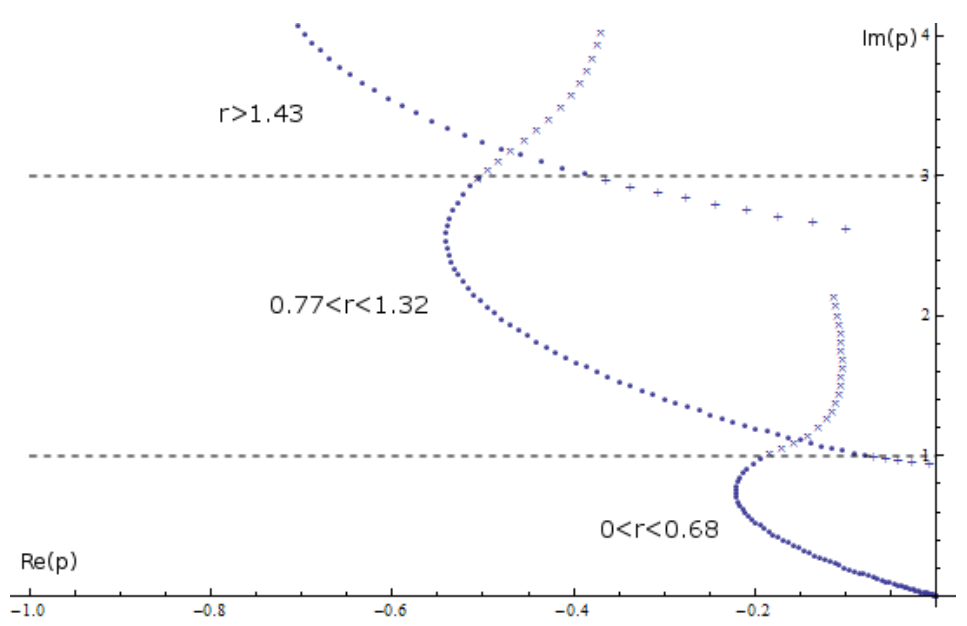

Figure 5. Position of resonances for different $r$. Dots are resonances for the usual branch, and " $\times$ " and "+" are those resonances continuing on the Riemann surface (they are not visible with the usual branch cut). The " $\times$ " and "+" curves in the middle are on different Riemann sheets.

$$
(\sqrt{-i} \sqrt{p}+1) \hat{\psi}(0, p)=r \hat{\psi}(0, p-i \omega)+r \hat{\psi}(0, p+i \omega)+\sqrt{-i} \sqrt{p} f(0, p)
$$

With a change of branch $\sqrt{p} \rightarrow-\sqrt{p}$ and changes of variables $r \rightarrow-r, f \rightarrow-f$, the above equation becomes

$$
(\sqrt{-i} \sqrt{p}-1) \hat{\psi}(0, p)=r \hat{\psi}(0, p-i \omega)+r \hat{\psi}(0, p+i \omega)+\sqrt{-i} \sqrt{p} f(0, p)
$$

which is identical to (9).

Therefore essentially all the theoretical results hold for this case as well. Note, however, that for small $r$ there is no resonance with the usual choice of branch (which corresponds to a different choice of branch in the potential barrier case, see Proposition 14).

For larger $r$, we expect the behavior of the wave function to be qualitatively similar to that with a delta potential well, since the contribution from the timeindependent part will be relatively insignificant compared to the time-dependent part. This is confirmed with the graph below plotted for different $r$ and $\omega=2$. We choose the usual branch for simplicity.

Acknowledgments. The author is grateful to O. Costin who introduced him to questions of the type addressed in the paper and gave him many valuable suggestions.

\section{REFERENCES}

[1] G. Gamow, Zur Quantentheorie de Atomkernes, Z. Phys. 51, 204-212 (1928).

[2] P. Garrido, S. Goldstein, J. Lukkarinen and R. Tumulka, Paradoxical Reflection in Quantum Mechanics arXiv:0808.0610

[3] E. Skibsted, Comm. Math. Phys. 104 no. 4, pp 591-604 (1986). 


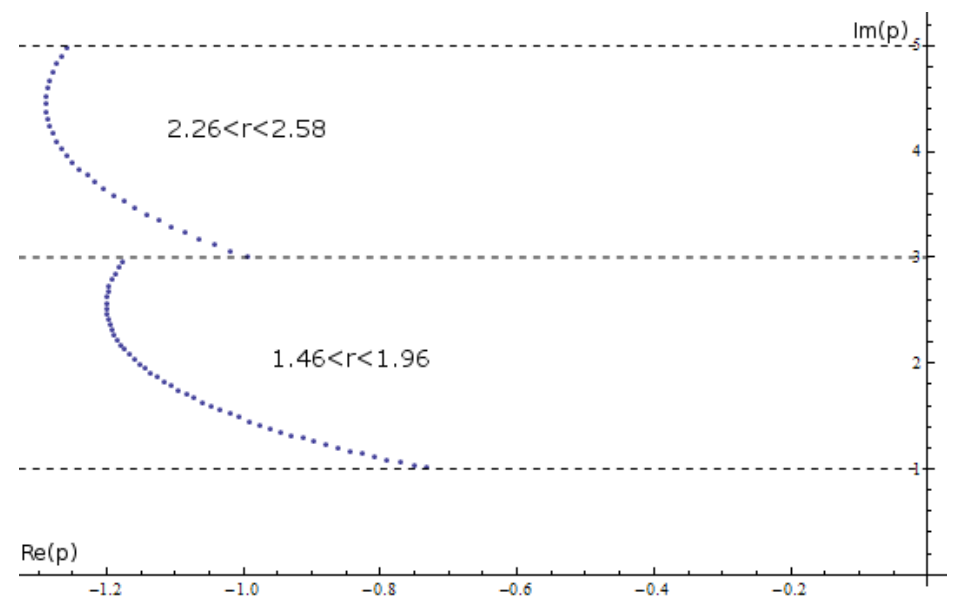

Figure 6. Position of resonances for different $r$ for the delta potential barrier.

[4] J. C. Wells, I. Simbotin, and M. Gavrila, Multistate High-Frequency Floquet Theory, Laser Physics, Vol. 7, No. 3, 1997, pp. 525-536.

[5] O. Costin, J. L. Lebowitz and A. Ronhlenno, On the complete ionization of a periodically perturbed quantum system, CRM Proceedings and Lecture Notes 27 pp 51-61 (2001)

[6] M Reed and B Simon, Methods of modern mathematical physics, Academic Press, New York (1972).

[7] O. Costin, R. D. Costin, J. L. Lebowitz, Time asymptotics of the Schröinger wave function in time-periodic potentials, J. Stat. Phys., special issue dedicated to Elliott Lieb on the occasion of his 70th birthday, 1-4 283-310 (2004).

[8] O. Costin, Asymptotics and Borel Summability C R C Press LLC, (2008).

[9] R. de la Madrid and M. Gadella, Amer. J. Phys. 70 no. 6, pp. 626-638, (2002).

[10] E. Kapuścik P. Szczeszek, The Physical Mechanism of Formation of Quantum Mechanical Gamow States, Foundations of Physics Letters, vol. 18, issue 6, pp. 573-580

[11] Multiphoton Ionization of Atoms, S. L. Chin and P. Lambropoulus, editors, Academic Press (1984).

[12] G Mainfray and G Manus, Multiphoton ionization of atoms Rep. Prog. Phys. 54 1333-1372 (1991) doi: 10.1088/0034-4885/54/10/002

[13] Gribakin, G. F.; Kuchiev, M. Yu. Multiphoton detachment of electrons from negative ions Physical Review A (Atomic, Molecular, and Optical Physics), Volume 55, Issue 5, May 1997, pp.3760-3771

[14] Dario Bambusi, Sandro Graffi, Time Quasi-periodic unbounded perturbations of the Schrödinger operators and KAM methods, Comm. Math. Phys. 219 n.2, 465-480 (2001)

[15] O. Costin, M. Huang, Gamow vectors and Borel summability, submitted

[16] O. Costin, M. Huang, Z. Qiu, Ionization in damped time-harmonic fields, submitted

Mathematics Department, The Ohio State University, Columbus, OH 43210 